\title{
Randomized Clinical Stroke Trials in 2007
}

\author{
Meheroz H. Rabadi*,1 and John P. Blass ${ }^{2}$ \\ ${ }^{I}$ Veterans Affairs Medical Center at Oklahoma University; ${ }^{2}$ Burke Medical Research Institute Weill Medical College of \\ Cornell University, USA
}

\begin{abstract}
This article reviews the randomized control trials (RCT's) that were published in 2007 of emerging pharmacotherapies in patients with acute ( $\leq 2$ weeks), sub-acute ( 2 to 12 weeks) and chronic $(\geq 12$ weeks) stroke. A Medline search generated 22 RCT's in stroke in the year 2007 in the English language. These trials were primarily efficacy studies. These included the role of statins (an anti-lipid agent) in reducing post-stroke morbidity and mortality, and decreasing the carotid atherosclerotic plaque in middle aged patients at increased risk of cardiovascular disease; glucose-potassiuminsulin infusion in hyperglyceamic acute stroke patients; pioglitazone (an anti-diabetic medication) to reduce recurrence of stroke in Type 2 diabetic patients; administration of intra-arterial urokinase (a thrombolytic agent) and the role of laser therapy in clot dissolution given that at present there is only one FDA approved thrombolytic agent ( $\mathrm{r}$ TPA); benefit of warfarin (an anticoagulant) in elderly patients with atrial fibrillation in the community; NXY (a free radical trapping agent) and minocycline both tested as neuroprotectants; and zoledronate (an intravenous bisphosphonate) to prevent loss of bone mineral density of the affected extremity, and finally the role of nicardipine (a Calcium channel blocker) in the prevention of vasospasm, and hydrocortisone to prevent hyponatraemia after sub-arachnoid hemorrhage. Finally the role of non-pharmacotherapy like stents for patient's with internal carotid artery dissection with tandem internal carotid and middle cerebral artery occlusion and in vertebral artery stenosis.
\end{abstract}

Keywords: Randomized control trials, stroke, rehabilitation.

\section{INTRODUCTION}

This review focuses on the clinical usefulness of commonly used pharmacological agents, readily available to clinicians caring for stroke patients that were assessed in clinical trials published in 2007.

\section{Hyperlipidaemia and Stroke}

Title: Statin treatment withdrawal in ischemic stroke: a controlled randomized study.

Pretreatment with statins has been shown to reduce brain injury in cerebral ischemia [1]. This randomized control study, investigated the influence of pretreatment statin and its subsequent withdrawal on outcome after acute ischemic stroke [2]. Of the 215 patients admitted within 24 hours of a hemispheric ischemic stroke, 89 patients on chronic statin treatment were randomly assigned either to statin withdrawal for the first 3 days after admission $(n=46)$ or to continue receiving atorvastatin $20 \mathrm{mg} /$ day $(\mathrm{n}=43)$. The primary outcome event was death or dependency (modified Rankin Scale [mRS] score > 2) at 3 months. Secondary outcome variables were early neurologic deterioration (END) and infarct volume at days 4 to 7 . In a secondary analysis, outcome variables were compared with the nonrandomized patients without previous statin therapy $(\mathrm{n}=126)$. Patients with statin withdrawal showed a higher frequency of $\mathrm{mRS}$ score $>$ 2 at the end of follow-up (60.0\% vs. $39.0 \%$; $\mathrm{p}=0.043)$, END $(65.2 \%$ vs. $20.9 \% ; \mathrm{p}<0.0001)$, and greater infarct volume

*Address correspondence to this author at the Veterans Affairs Medical Center, $921 \mathrm{NE} 13^{\text {th }}$ Street, Oklahoma City OK, USA; Tel: (405) 270- 0501 Extension 5298; Fax: (405) 270- 1504; E-mail: mhrabadi@gmail.com
$(74[45,126]$ vs. $26[12,70] \mathrm{mL} ; \mathrm{p}=0.002)$ compared with the non-statin-withdrawal group. Statin withdrawal was associated with a 4.66 (1.46 to 14.91)-fold increase in the risk of death or dependency, a 8.67 (3.05 to 24.63)-fold increase in the risk of END, and an increase in mean infarct volume of $37.63 \mathrm{~mL}$ (SE 10.01; $\mathrm{p}<0.001$ ) after adjusting for age and baseline stroke severity. Compared with patients without previous treatment with statins, withdrawal was associated with a 19 (1.96 to 184.09)-fold increase in the risk of END and an increase in mean infarct volume of $43.5 \mathrm{~mL}$ (SE 21.9; $\mathrm{p}=0.048$ ). The study concluded that statin withdrawal was associated with increased risk of death or dependency at 90 days. Hence, this treatment should be continued in the acute phase of ischemic stroke.

Title: Measuring Effects on Intima-Media Thickness: an Evaluation of Rosuvastatin (METEOR).

Atherosclerosis is often advanced before symptoms appear and it is unclear whether treatment is beneficial in middle-aged individuals with a low Framingham risk score (FRS) and mild to moderate subclinical atherosclerosis. This study assessed whether statin therapy could slow the progression and/or reduce carotid intima-media thickness (CIMT) over 2 years, as assessed with B-mode ultrasound for 12 carotid sites [3]. In this randomized, double-blind, international, placebo-controlled study 984 individuals were enrolled with age (mean, 57 years) as the only coronary heart disease risk factor or a 10-year FRS of less than 10\%, modest CIMT thickening $(1.2-<3.5 \mathrm{~mm})$ with mean CIMT for the common carotid artery sites $0.76 \pm 0.12 \mathrm{~mm}$ and elevated LDL cholesterol (mean, $154 \mathrm{mg} / \mathrm{dL}$ ). Participants received either a 40-mg dose of rosuvastatin $(\mathrm{n}=702)$ or placebo 
$(n=282)$. Among participants in the rosuvastatin group, the mean (SD) baseline LDL cholesterol level of 155 (24.1) $\mathrm{mg} / \mathrm{dL}$ declined to $78(27.5) \mathrm{mg} / \mathrm{dL}$, a mean reduction of $49 \%$ ( $\mathrm{P}<.001$ vs. placebo group). The change in maximum CIMT for the 12 carotid sites was -0.0014 (95\% CI, -0.0041 to 0.0014 ) for the rosuvastatin group vs. 0.0131 (95\% CI, $0.0087-0.0174)$ for the placebo group $(\mathrm{P}<.001)$. The maximum change in CIMT for the rosuvastatin group was $0.0038(95 \% \mathrm{CI},-0.0064$ to -0.0013$) \mathrm{mm} / \mathrm{y}$ for the common carotid artery sites $(\mathrm{P}<.001),-0.0040(95 \% \mathrm{CI},-0.0090$ to $0.0010)$ for the carotid bulb sites $(\mathrm{P}<.001)$, and $0.0039(95 \%$ CI, -0.0009 to 0.0088$)$ for the internal carotid artery sites $(\mathrm{P}$ $=.02$ ). The change in mean CIMT for the rosuvastatin group for the common carotid artery sites was 0.0004 (95\% CI, 0.0011 to 0.0019$) \mathrm{mm} / \mathrm{y}(\mathrm{P}<.001)$. All p-values were vs. placebo group. Overall, rosuvastatin was well tolerated with infrequent serious adverse cardiovascular events over 2 years. In middle-aged adults with an FRS of less than $10 \%$ and evidence of subclinical atherosclerosis, rosuvastatin significant reduced the rate of progression of maximum CIMT over 2 years vs. placebo. However, long-term trial is needed to determine the clinical implications of these findings.

\section{Treatment of Diabetes Mellitus and Stroke}

Title: Effects of pioglitazone in patients with type 2 diabetes with or without previous stroke: results from PROactive (PROspective pioglitAzone Clinical Trial In macroVascular Events [4].

Diabetes is an important risk factor for stroke. This prospective, double-blind study randomized 5238 patients with type 2 diabetes and a history of macrovascular disease to either pioglitazone (titrated to $45 \mathrm{mg}$ ) or placebo, in addition to continuing their diabetes and cardiovascular medications [4]. This study evaluated the risk of stroke and other cardiovascular outcomes in patients with $(n=984)$ and without $(n=4254)$ prior stroke. In patients with previous stroke (486 in the pioglitazone group and 498 in the placebo group), there was a trend favoring the pioglitazone for the primary end points: all-cause death, nonfatal myocardial infarction, acute coronary syndrome, and cardiac intervention (including coronary artery bypass graft or percutaneous coronary intervention), stroke, major leg amputation, or bypass surgery or leg revascularization (hazard ratio[HR] $=0.78$, event rate $=20.2 \%$ pioglitazone vs. $25.3 \%$ placebo; 95\% $\mathrm{CI}=0.60-1.02 ; \mathrm{P}=0.07)$. Similar results were obtained for secondary end points: all-cause death, nonfatal myocardial infarction, or nonfatal stroke $(\mathrm{HR}=0.78$, event rate $=15.6 \%$ pioglitazone vs. $19.7 \%$ placebo; $95 \%$ $\mathrm{CI}=0.58-1.06 ; \quad \mathrm{P}=0.11)$. Pioglitazone reduced fatal or nonfatal stroke $(\mathrm{HR}=0.53$, event rate $=5.6 \%$ pioglitazone vs. $10.2 \%$ placebo; $95 \% \quad \mathrm{CI}=0.34-0.85 ; \quad \mathrm{P}=0.008) \quad$ and cardiovascular death, nonfatal myocardial infarction, or nonfatal stroke $(\mathrm{HR}=0.72$, event rate $=13.0 \%$ pioglitazone vs. $17.7 \%$ placebo; $95 \% \mathrm{CI}=0.52-1.00 ; \mathrm{P}=0.0467)$. Higher event rates were observed in patients with prior stroke compared with those without prior stroke. In patients without prior stroke no treatment benefit was observed. Thus in the subgroup analysis of this study, pioglitazone was effective in secondary not in primary stroke prevention in patients with type 2 diabetes.
Title: Glucose-potassium-insulin infusions in the management of post-stroke hyperglycaemia: the UK Glucose Insulin in Stroke Trial (GIST-UK).

Hyperglycaemia after an acute stroke is a common finding and has been associated with an increased risk of death. This study set out to determine whether treatment with glucose-potassium-insulin (GKI) infusions to maintain euglycaemia immediately after the acute event reduces death at 90 days [5]. Patients presenting within $24 \mathrm{~h}$ of stroke onset and with admission plasma glucose concentration between 6.0$17.0 \mathrm{mmol} / \mathrm{L}$ were randomly assigned to receive variabledose-insulin GKI (intervention) or saline (control) as a continuous intravenous infusion for $24 \mathrm{~h}$. The aim was to maintain capillary glucose at 4-7 $\mathrm{mmol} / \mathrm{L}$ in the GKI group, with no glucose intervention in the control group. The primary outcome was death at 90 days, and the secondary endpoint was avoidance of death or severe disability at 90 days. The trial was powered to detect a mortality difference of $6 \%$ (sample size 2355), with $83 \%$ power, at the $5 \%$ two-sided significance level. This study was stopped after 933 patients were recruited due to slow enrollment. For the intention-totreat data, there was no significant reduction in mortality at 90 days (GKI vs. control: odds ratio $1.14,95 \%$ CI 0.86-1.51, $\mathrm{p}=0.37)$. There were no significant differences for secondary outcomes. In the GKI group, overall mean plasma glucose and mean systolic blood pressure were significantly lower than in the control group (mean difference in glucose 0.57 $\mathrm{mmol} / \mathrm{L}, \mathrm{p}<0.001$; mean difference in blood pressure 9.0 $\mathrm{mmHg}, \mathrm{p}<0.0001)$. The study concluded that despite significant reduction in the plasma glucose concentrations and blood pressure in the GKI group, these interventions did not reduce death or disability rates. Thus maintaining euglycaemia by GKI infusion may have a very small effect in reducing death or disability, given that it could not be seen in $>900$ subjects studied.

\section{Thrombolytics and Stroke:}

Title: A pilot study of dual treatment with recombinant tissue plasminogen activator and uric acid in acute ischemic stroke.

Uric acid (UA) has been shown to increase the neuroprotective effects of recombinant tissue plasminogen activator (rt-PA) in experimental ischemia [6]. In patients with stroke, increased UA levels have been linked to better stroke recovery [7], but the clinical safety of dual administration of UA and rt-PA is unknown. This was assessed in a double-blind study [8]. Patients were randomized to an intravenous solution of $500 \mathrm{~mL}$ of $5 \%$ mannitol/0.1\% lithium carbonate (control group, $n=8)$ or 500 or $1000 \mathrm{mg}$ of UA $(n=16)$. Safety end points at day 90, lipid peroxidation (serum malondialdehyde), and serum kinetics of UA were established. Twentyfour patients with stroke were treated with rt-PA within mean (SD) 133 (35) minutes of clinical onset (admission National Institutes of Health Stroke Scale score mean [SD] 11 [7], age 71 [10.6] years, 71\% males). Levels of UA decreased in the control group and increased for 24 hours in the high dose UA group, which also had lower levels of malondialdehyde at day 5. Mortality (12.5\%), symptomatic central nervous system bleeding $(0 \%)$, and outcome at day 90 were similar in the 2 treatment arms; one patient in the high-dose UA group had a mild gouty episode. Administration of UA appeared to be safe, decreased lipid peroxidation, 
and prevented an early fall of UA in serum in patients treated with rt-PA within 3 hours of stroke onset. However, there were no beneficial clinical effects between the 2 groups. The clinical efficacy of dual administration of exogenous UA and rt-PA needs further investigation in a larger acute stroke trial.

Title: The NeuroThera Effectiveness and Safety Trial-1 (NEST-1).

This study evaluated the safety and effectiveness of the NeuroThera Laser System (NTS) to improve 90-day outcomes in ischemic stroke patients treated within 24 hours from stroke onset. The NeuroThera Laser System uses infrared laser technology that involves photobiostimulation (which is increased adenosine triphosphate [ATP] formation on stimulation of the copper center $\mathrm{Cu}_{\mathrm{A}}$ of the cytochrome $\mathrm{c}$ oxidase, the primary mitochondrial chromophore to $808 \mathrm{~nm}$ energy delivered by NTS) and has shown significant and sustained beneficial effects in animal models of ischemic stroke [9]. In this prospective, intention-to-treat, multicenter, international, double-blind, trial, 120 acute ischemic stroke patients were randomized in a 2:1 ratio, with 79 patients in the active treatment group and 41 in the sham (placebo) control group [10]. The patients had baseline stroke severity scores of 7 to 22, as measured by National Institutes of Health Stroke Scale (NIHSS). Patients who received tissue plasminogen activator were excluded. The primary outcome measure identified as successful treatment, was defined as a complete recovery at day 90 (NIHSS 0 to 1 ), or a decrease in NIHSS score of at least 9 points (day 90 versus baseline), and was tested as a binary measure (bNIH). Secondary outcome measures included modified Rankin Scale mRS, Barthel Index, and Glasgow Outcome Scale at 90 days after treatment. Primary statistical analyses performed with the Cochran-Mantel-Haenszel rank test ; were stratified by baseline NIHSS score or by time to treatment for the bNIH and mRS. The mean time to treatment was $>16$ hours (median time to treatment 18 hours for active and 17 hours for control). Time to treatment ranged from 2 to 24 hours. More patients $(70 \%)$ in the active treatment group had successful outcomes than did controls (51\%), as measured prospectively on the bNIH $(\mathrm{P}=0.03$ stratified by severity and time to treatment; $\mathrm{P}=0.048$ stratified only by severity). Similarly, more patients (59\%) had successful outcomes than did controls $(44 \%)$ as measured at 90 days as a binary mRS score of 0 to $2(\mathrm{P}=0.03$ stratified by severity and time to treatment; $\mathrm{P}=0.04$ stratified only by severity). Also, more patients in the active treatment group had successful outcomes than controls as measured by the change in mean NIHSS score from baseline to 90 days ( $\mathrm{P}=0.02$ stratified by time to treatment) and the full mRS ("shift in Rankin") score ( $\mathrm{P}=0.02$ stratified by severity and time to treatment; $\mathrm{P}=0.03$ stratified only by severity). Mortality rates and serious adverse events (SAEs) did not differ significantly (8.9\% and $25.3 \%$ for active $9.8 \%$ and $36.6 \%$ for control, respectively, for mortality and SAEs). The NEST-1 study indicated that infrared laser therapy to be a safe and effective treatment for ischemic stroke in humans when initiated within 24 hours of stroke onset. A larger confirmatory trial to determine safety and effectiveness more conclusively is warranted.

Title: Intra-arterial infusion of urokinase within 6 hours of middle cerebral artery stroke: the Middle cerebral artery
Embolism Local fibrinolytic intervention Trial (MELT Japan).

The MELT Japan was organized to determine the safety and clinical efficacy of intra-arterial infusion of urokinase (UK) within 6 hours of stroke onset [11]. Patients with ischemic stroke presenting within 6 hours of onset and displaying occlusions of the M1 or M2 portion of the middle cerebral artery on carotid angiography were randomized to the UK or control groups. The modified Rankin Scale (mRS), National Institutes of Health Stroke Scale, and Barthel Index were used to assess clinical outcome. The Independent Monitoring Committee recommended stopping the trial after approval of intravenous infusion of recombinant tissue plasminogen activator in Japan. A total of 114 patients underwent randomization, 57 patients in each group. Background characteristics were comparable between the 2 groups. The primary end point of favorable outcome (mRS 0 to 2) at 90 days was numerically higher in the UK-treated than in the control group (49.1\% and $38.6 \%$, OR: $1.54,95 \%$ CI: 0.73 to 3.23$)$ but was not statistical $(\mathrm{P}=0.345)$. Excellent functional outcome (mRS 0 to 1 ) at 90 days, a preplanned secondary end point, was more frequent in the UK than in the control group $(42.1 \%$ and $22.8 \%, \mathrm{P}=0.045$, OR: 2.46 , 95\% CI: 1.09 to 5.54$)$. There were also more patients with National Institutes of Health Stroke Scale 0 or 1 at 90 days in the UK group than the control group ( $\mathrm{P}=0.017)$. The 90 -day cumulative mortality was $5.3 \%$ in the UK group and $3.5 \%$ in the control group $(\mathrm{P}=1.000)$, and intracerebral hemorrhage within 24 hours of treatment occurred in $9 \%$ and $2 \%$, respectively $(\mathrm{P}=0.206)$. Since this trial was stopped early it was not sufficiently powered to provide more definitive conclusions. The primary end point did not reach statistical significance. However, the secondary analyses showed that intra-arterial thrombolysis to have the potential to increase excellent functional outcome. The MELT study design paralleled the PROACT II study [12]. However, in the PROACT II study the similar primary end point favored the UK group, which was not reported in the MELT study. There are several reasons suggested for this failure to show efficacy in this study. First, because this trial was terminated prematurely, the number of patients enrolled was too low to detect any difference between the UK and control groups in the primary end point. Second, there were $38.6 \%$ of patients with mRS score 0 to 2 in the control group. Thus the patients in the MELT Japan study had milder strokes. Likewise, the median NIHSS score was lower than in the PROACT studies. Finally, the MELT Japan prohibited mechanical clot disruption except for the guide-wire technique to examine the effect of UK itself. The re-canalization rate in this study was $73.7 \%$ which was better than $66 \%$ reported in the PROACT II study, which prohibited any mechanical procedure [12]. Therefore, mechanical fibrinolysis in this study may have be one of the reasons for the present favorable re-canalization rate.

\section{Antiplatelets and Stroke}

Title: Medium intensity oral anticoagulants versus aspirin after cerebral ischemia of arterial origin [The European/Australasian Stroke Prevention in Reversible Ischemia Trial (ESPRIT)]: a randomized controlled trial.

Oral anticoagulants are better than aspirin for secondary prevention after myocardial infarction and after cerebral 
ischemia in patients with non-rheumatic atrial fibrillation [13]. ESPRIT aimed to determine whether medium intensity of oral anticoagulation with was more effective than aspirin in preventing future vascular events in patients with transient ischemic attack or minor stroke of presumed arterial origin [14]. In this international, multi-centre open label trial, patients within 6 months after a transient ischemic attack or minor stroke of presumed arterial origin were randomly assigned to either anticoagulants (target INR range 2.0-3.0; $\mathrm{n}=536)$ or aspirin (30-325 mg daily; $\mathrm{n}=532)$. The primary outcome was the composite of death from all vascular causes, non-fatal stroke, non-fatal myocardial infarction, or major bleeding complication, whichever occurred first. In a post hoc analysis anticoagulants were compared with the combination of aspirin and dipyridamole (200 mg twice daily). The primary analysis was by intention to treat. The anticoagulant versus aspirin comparison of ESPRIT was prematurely ended because ESPRIT had previously showed that that the combination of aspirin and dipyridamole was superior to aspirin alone. ESPRIT had a mean follow-up of 4.6 years (SD 2.2). The mean achieved INR was 2.57 (SD $0.86)$. A primary outcome event occurred in $99(19 \%)$ patients on anticoagulants and in $98(18 \%)$ patients on aspirin (hazard ratio [HR] $1.02,95 \%$ CI $0.77-1.35$ ). The HR for ischemic events was $0.73(0.52-1.01)$ and for major bleeding complications was $2.56(1.48-4.43)$ in the anticoagulation group. The HR for the primary outcome event comparing anticoagulants with the combination treatment of aspirin and dipyridamole was $1.31(0.98-1.75)$. This study showed that medium intensity oral anticoagulation (target INR range 2.03.0) was equally effective as aspirin for secondary prevention after transient ischemic attack or minor stroke of arterial origin. Increased bleeding complications in the oral anticoagulation group offset its protective effect against ischemic events.

\section{Treatment of Atrial Fibrillation and Stroke}

Title: Warfarin versus aspirin for stroke prevention in an elderly community population with atrial fibrillation (the Birmingham Atrial Fibrillation Treatment of the Aged Study. [BAFTA]): a randomised controlled trial.

Warfarin reduces recurrent stroke risk in patients with atrial fibrillation [15]. Whether this benefit outweighs the increased risk of bleeding in elderly patients is unknown. This study assessed whether warfarin reduced risk of major stroke, arterial embolism, or other intracranial hemorrhage compared with aspirin (ASA) in elderly patients [16]. Some 973 patients aged 75 years or over (mean age 81.5 years, SD 4.2) with atrial fibrillation were recruited from primary care and randomly assigned to either warfarin (target international normalized ratio $2-3$ ) or aspirin (75 mg per day). Follow-up was for a mean of 2.7 years (SD 1.2). The primary endpoint was fatal or disabling stroke (ischemic or hemorrhagic), intracranial hemorrhage, or clinically significant arterial embolism. Analysis was by intention to treat. There were 24 primary events (21 strokes, two intracranial hemorrhages, and one systemic embolus) in people assigned to the warfarin group and 48 primary events (44 strokes, one intracranial hemorrhage, and three systemic emboli) in people assigned to aspirin (yearly risk $1.8 \%$ vs $3.8 \%$, relative risk $0.48,95 \%$ CI $0.28-0.80, \mathrm{p}=0.003$; absolute yearly risk reduction $2 \%$,
95\% CI 0.7-3.2). The annual risk of extra-cranial hemorrhage was $1.4 \%$ (warfarin) versus $1.6 \%$ (aspirin). This study suggests anticoagulation therapy benefits people aged over 75 who have atrial fibrillation, unless there are contraindications to its use or the patient decides that the hemorrhagic risk outweighs its benefit.

\section{Neuroprotection and Stroke}

Title: Cerebral Hematoma and NXY Treatment trial (CHANT).

NXY-059 is a free radical-trapping agent which neuroprotects by preventing brain tissue oxidation in acute ischemic stroke. This study investigated the safety of NXY059 in patients with intracerebral hemorrhage (ICH) [17]. Some 607 patients within 6 hours of acute ICH received $2270 \mathrm{mg}$ intravenous NXY-059 over 1 hour and then up to $960 \mathrm{mg} / \mathrm{h}$ over the subsequent 71 hours $(\mathrm{n}=300)$, or matching placebo $(n=303)$, in addition to standard care. The primary outcomes for safety were: the mortality and the frequency of adverse clinical events. Other tests for adverse events included a change from baseline for serum potassium, intracranial hematoma volume on neuroimaging, and electrocaardiographic changes during infusion period. The overall distribution of disability scores on the modified Rankin Scale $(\mathrm{mRS})$ and the Barthel index were also studied. Treatment groups were well matched for prognostic variables including Glasgow Coma Scale, risk factors, and age. The mean National Institute of Health Stroke Scale score on admission was 14 in both groups. The baseline hemorrhage volume was $22.4 \pm 20.1 \mathrm{~mL}$ in the NXY-059 group and $23.3 \pm 22.8 \mathrm{~mL}$ in the placebo group (mean $\pm \mathrm{SD}$ ). Most hemorrhages were related to hypertension or anticoagulant use. Mortality was similar in both groups: $20.3 \%$ for NXY-059 and $19.8 \%$ for placebo-treated patients. The incidence of adverse event was the same for both groups; however, the proportion of serious adverse events was slightly higher in the NXY-059 group. Serum potassium fell transiently in both groups, but was lower in the NXY-059 group. No differences in 3-month functional disability, or neurological deficit scores were noted. The odds ratio for an improved outcome in 3-month mRS scores in the NXY-059 group was 1.01 (95\% CI 0.75, 1.35). NXY-059 given within 6 hours of acute ICH was to found a good safety and tolerability profile, with no adverse effect on clinical outcomes but also no benefits.

Title: Stroke-Acute ischemic NXY Treatment (SAINT II) Trial Investigator.

The free-radical-trapping agent NXY-059 had shown promise as a neuroprotectant in the Stroke-Acute Ischemic NXY Treatment I (SAINT I) trial [18] by reducing disability when given to patients who had acute ischemic stroke. The current study sought to confirm this efficacy in a second, larger trial [19]. Some 3,306 patients with acute ischemic stroke were randomized in a double-blind trial to receive a 72-hour infusion of intravenous NXY-059 or placebo within 6 hours after the onset of stroke symptoms. The primary end point was the distribution of disability scores at 90 days on the modified Rankin scale (mRS). The National Institute of Health Stroke Scale and the Barthel Index were secondary end points. The efficacy analysis was based on 3195 patients. Demographic variables were well balanced between the treatment groups. The distribution of scores on the mRS 
did not differ between the NXY-059 treated (1588 patients) and the placebo (1607) group; $\mathrm{P}=0.33$ by the CochranMantel-Haenszel test; odds ratio for limiting disability, 0.94; $95 \%$ confidence interval $[\mathrm{CI}], 0.83$ to 1.06 ). Analysis of categorized scores on the modified Rankin scale confirmed the lack of benefit: the odds ratio for the three stratified modified Rankin scale scores of 0 to 1 versus 2 to 3 versus 4 to 6 was 0.92 (95\% CI, 0.80 to 1.06). There was no evidence of efficacy for any of the secondary end points. Mortality was equal in the two groups, and adverse-event rates were similar. Among patients treated with alteplase, there was no difference between the NXY-059 group and the placebo group in the frequency of symptomatic or asymptomatic hemorrhage. Thus NXY-059 was ineffective for the treatment of acute ischemic stroke within 6 hours after the onset of symptoms. This study like several earlier phase III trials of neuroprotection has failed to live up to the expectations created by preclinical testing in animal models. Several reasons have been cited for these disappointing human results: a) animal models may not mimic human ischemic stroke; b) preclinical trials have administered the drug prior to or within a few minutes of lesion being made; c) lack of randomization; d) lack of in vitro efficacy in human tissue using cell cultures or brain slices; e) lack of in vivo studies of neuroprotectants distribution in the normal and ischemic brain; and f) lack of use of imaging techniques in phase II and III neuroprotectant studies to identify the extent of the pneumbra, its target site.

Title: Minocycline treatment in acute stroke: an openlabel, evaluator-blinded study.

Minocycline has a neuroprotective effect in ischemic animal models [20]. This study assessed the effect of minocycline treatment in human acute ischemic stroke [21]. In this open-label, evaluator-blinded study, Minocycline in a dosage of $200 \mathrm{mg}$ was administered orally for 5 days starting within 6 to 24 hours after onset of stroke. The outcome measures were: NIH Stroke Scale (NIHSS), modified Rankin Scale (mRS), and Barthel Index (BI). The primary objective was to compare changes from baseline to day 90 in NIHSS in the minocycline group vs. placebo. Of the 152 patients enrolled in the study, 74 received minocycline treatment and 77 placebo. NIHSS and mRS were significantly lower and BI scores were significantly higher in minocycline-treated patients. This pattern was already apparent on day 7 and day 30 of follow-up. Deaths, myocardial infarctions, recurrent strokes, and hemorrhagic transformations during follow-up were similar in both groups. This study suggests minocycline to be of potential functional benefit in acute ischemic stroke. Despite minocycline being effective in ischemic stroke patients, the small sample size and open-label study indicate the need for a large randomized control trial.

\section{Surgery for Stroke}

Title: Long-term outcome after angioplasty and stenting for symptomatic vertebral artery stenosis compared with medical treatment in the Carotid and Vertebral Artery Transluminal Angioplasty Study (CAVATAS): a randomized trial.

The long-term outcome of endovascular intervention compared with best medical management of patients with symptomatic vertebral artery stenosis is not known. In this international, multi-center study, 16 patients with symptomatic vertebral artery stenosis were randomized in equal proportions to receive endovascular therapy (balloon angioplasty or stenting) or best medical treatment alone [22]. An independent neurologist followed up the patients for 8 years. Endovascular intervention was technically successful in all 8 patients and there were no deaths or strokes in any arterial territory within the first 30 days. During a mean follow-up period of 4.7 years, no patient in either treatment group experienced a vertebrobasilar territory stroke, but 3 patients in each treatment arm died of myocardial infarction or carotid territory stroke, and 1 endovascular patient had a nonfatal carotid territory stroke. The study concluded that patients with vertebral artery stenosis were more likely to have carotid territory stroke and myocardial infarction during follow-up than to have recurrent vertebrobasilar stroke. This trial failed to show a benefit of endovascular treatment for vertebral artery stenosis. The reason for lack of benefit of endovascular treatment may mainly be due to the small numbers of patients included in the study. Larger randomized trials would be required to determine whether vertebral artery stenting is justified in patients at higher risk of vertebrobasilar stroke. This study highlights that in patients with vertebral artery stenosis the focus should be on global reduction of vascular risk factors which in turn would prevent further carotid territory stroke and myocardial infarction.

Title: Stent-assisted endovascular thrombolysis versus intravenous thrombolysis in internal carotid artery dissection with tandem internal carotid and middle cerebral artery occlusion.

Spontaneous dissection of the internal carotid artery (ICA) is one of the main causes of ischemic stroke in young patients, and anticoagulation is its recommended treatment [23]. Tandem ICA and middle cerebral artery occlusions independently predict poor outcome after intravenous thrombolysis. Recently recanalization of ICA dissection by stent-assisted angioplasty has recently been proposed when anticoagulation fails to prevent a new ischemic event. Consecutive patients presenting within 3 hours of symptom onset with tandem internal carotid and middle cerebral artery occlusion with ICA dissection, who were eligible for intravenous thrombolysis, were randomized to either endovascular stent-assisted thrombolysis $(\mathrm{n}=6)$ or intravenous recombinant tissue-type plasminogen activator (rtPA) when an endovascular therapist was unavailable $(n=4)$ [24]. National Institutes of Health Stroke Scale (NIHSS) scores were obtained at baseline and after 24 hours. The modified Rankin Scale score (mRS) was used to assess outcomes at 3 months. Arterial recanalization was assessed by magnetic resonance imaging. The mean NIHSS scores before treatment were high (17 and 16, respectively) in the 2 groups. In the endovascular group, all patients achieved middle cerebral artery recanalization with subsequent dramatic improvement versus only 1 patient with middle cerebral artery recanalization in the intravenous rtPA group. At 3 months, 4 patients in the endovascular group had a favorable outcome $(\mathrm{mRS}$ score $=0)$. In the intravenous rtPA group, 3 patients had a poor outcome (mRS score $\geq 3$ ). Thus endovascular stent-assisted thrombolysis was found to be a promising treatment option in tandem internal carotid and middle cerebral artery occlusion with ICA dissection and compared favorably to intravenous rtPA alone. 
Unfortunately, the small numbers of individuals studied in these tests of surgical interventions for stroke make confident conclusions impossible, despite the potential interest of these approaches.

\section{Stroke and Subarachnoid Hemorrhage (SAH)}

Title: Effect of nicardipine prolonged-release implants on cerebral vasospasm and clinical outcome after severe aneurysmal subarachnoid hemorrhage (SAH): a prospective, randomized, double-blind phase IIa study.

This study investigated the efficacy of nicardipine prolonged-release implants (NPRIs) on cerebral vasospasm and clinical outcome after severe subarachnoid hemorrhage (SAH) [25]. Thirty-two patients with severe SAH undergoing aneurysm clipping within 48 hours at a single center were randomized: 16 patients received NPRIs implanted into the basal cisterns in direct contact to the exposed proximal blood vessels; in 16 control patients, the basal cisterns were opened and washed out only without leaving implants. Angiography was performed preoperatively and at day $8 \pm 1$. Computed tomography imaging analyzed the incidence of territorial infarcts unrelated to surgery. Clinical outcome was assessed using the modified Rankin (mRS) and National Institute of Health Stroke (NIHSS) scales. The incidence of angiographic vasospasm in proximal vessel segments was significantly reduced after implantation of NPRIs (73\% control versus 7\% NPRIs). Computed tomography scans revealed a lower incidence of delayed ischemic lesions (47\% control versus $14 \%$ NPRIs). The NPRI group demonstrated more favorable mRS and NIHSS scales as well as a significantly fewer deaths (38\% control versus 6\% NPRIs). Thus NPRIs implants reduced the incidence of cerebral vasospasm and delayed ischemic deficits and improved clinical outcome after severe $\mathrm{SAH}$.

Title: A randomized controlled trial of hydrocortisone against hyponatremia in patients with aneurysmal subarachnoid hemorrhage.

Hyponatremia is common after aneurysmal subarachnoid hemorrhage (SAH). This is caused by the cerebral salt wasting syndrome, which induces osmotic diuresis and decreases blood volume, contributing to symptomatic cerebral vasospasm (SCV). Hypervolemic therapy in the presence of fludrocortisones has been effective in preventing SCV than by itself alone [26]. Seventy-one SAH patients were randomly assigned after surgery to treatment with either a placebo $(n=36)$ or $1200 \mathrm{mg} / \mathrm{d}$ of hydrocortisone $(\mathrm{n}=35)$ within 72 hours for 10 days and tapered thereafter [27]. Both groups underwent hypervolemic therapy. The primary end point was the prevention of hyponatremia. Hydrocortisone prevented excess sodium excretion $(\mathrm{P}=0.04)$ and urine volume $(\mathrm{P}=0.04)$. Hydrocortisone maintained the targeted serum sodium level throughout the 14 days $(\mathrm{P}<0.001)$, and allowed for lower sodium and fluid $(\mathrm{P}=0.007)$ supplementation. Hydrocortisone kept the plasma osmolarity normal $(\mathrm{P}<0.001)$. SCV occurred in 9 patients (25\%) in the placebo group and in $5(14 \%)$ in the hydrocortisone group. Thus hydrocortisone overcame excess natriuresis and prevented hyponatremia. Although no difference in SCV was found, hydrocortisone supported hypervoluemic therapy was more effective than hypervolemic therapy alone in treating SAH induced hyponatremia.
Title: Care management of poststroke depression: a randomized, controlled trial. Poststroke depression is a prevalent and disabling disorder.

This study determined the effectiveness of the "ActivateInitiate-Monitor" care management program for the treatment of poststroke depression [28]. The "Activate-InitiateMonitor" is a care management program that comprises of "Activation" making the patient recognize his depression symptoms and accept treatment, "Initiation" of making the patient take his antidepressant medication, and "Monitoring" to make adjustments to his antidepressant medication. Thus this was a proactive intervention in the management of poststroke depression. In this prospective, randomized, outcomeblinded trial of 188 ischemic stroke survivors identified at the time of admission to one of 4 Indianapolis hospitals, depression screening and enrollment occurred between 1 and 2 months post-stroke. Usual care subjects $(\mathrm{n}=93)$ received nondepression-related education and were prescribed antidepressants at the discretion of their provider. The primary outcome measure was depression response, defined as a Hamilton Depression Inventory score $<8$ (remission) or a decrease from baseline of at least $50 \%$ at 12 weeks. The intervention and usual care groups had similar key baseline measures. Both depression response (51\% versus $30 \%$, $\mathrm{P}=0.005$ ) and remission ( $39 \%$ versus $23 \%, \mathrm{P}=0.01$ ) occurred more frequently in the Activate-Initiate-Monitor intervention than in the usual care group. This difference in depression scores was present by 6 weeks and persisted through the 12week assessment. Serious adverse events did not differ between the 2 groups. The Activate-Initiate-Monitor care management model was more effective than usual care in improving depression outcome measures in patients with poststroke depression.

Title: Motivational interviewing early after acute stroke: a randomized, controlled trial.

The purpose of this study was to determine whether motivational interviewing, a patient-centered counseling technique, can benefit patient's mood 3 months after stroke [29]. In this single-center, open, randomized, controlled trial on a stroke unit, 411 consecutive patients who were over 18 years of age and who did not have severe cognitive and communication problems were enrolled with 28 days of their acute stroke. Patients in the intervention group received 4 individual, weekly sessions of motivational interviewing with a trained therapist in addition to usual stroke care. The primary outcome was the proportion of patients with normal mood at 3 months poststroke measured by the 28 -item General Health Questionnaire (normal, <5; low > or=5) using a mailed questionnaire. Eighty-one of 207 (39.1\%) patients in the control group and 100 of $204(49.0 \%)$ patients in the intervention group had normal mood at follow up. A significant benefit of motivational interviewing over usual stroke care (OR: 1.60, 95\% CI: 1.04 to $2.46, \mathrm{P}=0.03$ ) was found, suggesting that motivational interviewing leads to an improvement in patients' mood 3 months after stroke.

\section{Stroke and Bone Loss}

Title: A single infusion of zoledronate prevents bone loss after stroke.

Hip fractures are common in stroke patients due to limited mobility leading to disuse osteoporosis. Patients with 
moderate stroke severity at the time of hospital discharge have the most fractures. This proof-of-concept study evaluated the efficacy of a single infusion of zoledronate, an intravenous bisphosphonate, in preserving hip bone density after stroke [30]. In this double-blind, placebo-controlled, clinical trial, 27 hemiplegic patients (6 women, 21 men) within 35 days of acute stroke were randomized to receive 4 $\mathrm{mg}$ of intravenous zoledronate $(\mathrm{n}=14)$ or placebo $(\mathrm{n}=13)$. Both groups received calcium and vitamin D supplements. The primary outcome measure was the change in bone mineral density (BMD) at the hemiplegic hip during the year of investigation measured by dual energy x-ray absorptiometry (Lunar Prodigy). Mean total hip BMD was unchanged in the hemiplegic hip of the zoledronate group (mean $0.0 \%$ change), whereas in the placebo group the total hip BMD decreased by $-5.5 \%$, with the greatest bone loss observed in the trochanteric subregion (mean, $-8.1 \%$ ). On the unaffected side the mean change in total hip BMD was $+1.0 \%$ with zoledronate versus a mean change of $-2.7 \%$ without. Repeated measures ANOVA confirmed the significance of the differences between groups at both hips (hemiplegic, $\mathrm{P}<0.001$; unaffected, $\mathrm{P}=0.002$ ). The treatment was generally well tolerated. The study concluded that after a single infusion of zoledronate, patients with moderately severe strokes were protected from the harmful effects of hemiplegia on hip bone density for at least 1 year. Neither group of patients had any falls or fractures recorded during the study period.

\section{Stroke and Deep Vein Thrombosis}

Title: Prevention of venous thromboembolism after acute ischemic stroke (PREVAIL Study).

Low molecular weight heparin or unfractionated heparin is recommended for prophylaxis of venous thromboembolism in patients with acute ischemic stroke. This study aimed to compare which treatment regimen - enoxaparin or unfractionated heparin - was better for patients with acute ischemic stroke [31]. Some 1762 patients with acute ischemic stroke who were unable to walk unassisted were randomly assigned within $48 \mathrm{~h}$ of symptoms to receive either enoxaparin $40 \mathrm{mg}$ subcutaneously once daily or unfractionated heparin $5000 \mathrm{U}$ subcutaneously every $12 \mathrm{~h}$ for 10 days (range 6-14). Patients were stratified by National Institutes of Health Stroke Scale (NIHSS) score (severe stroke $\geq 14$, less severe stroke $<14)$. The primary efficacy endpoint was the composite of symptomatic or asymptomatic deep vein thrombosis, symptomatic pulmonary embolism, or fatal pulmonary embolism. Primary safety endpoints were: symptomatic intracranial hemorrhage, major extracranial hemorrhage, and all-cause mortality. Enoxaparin $(n=666)$ reduced the risk of venous thromboembolism by $43 \%$ compared with unfractionated heparin $(\mathrm{n}=669)(68[10 \%]$ vs. 121 [18\%]; relative risk $0.57,95 \%$ CI $0.44-0.76, \mathrm{p}=0.0001$; difference $7.9 \%,-11.6$ to -4.2$)$; this reduction was consistent for patients with an NIHSS score of 14 or more (26 [16\%] vs. 52 [30\%]; $\mathrm{p}=0.004)$ or less than 14 (42 [8\%] vs. 69 [14\%]; $\mathrm{p}=0.004)$. The occurrence of any bleeding was similar with enoxaparin $(69[8 \%])$ or unfractionated heparin $(71[8 \%]$; $\mathrm{p}=0.83$ ). The frequency of symptomatic intracranial and major extracranial hemorrhage was small and similar between groups (enoxaparin 11 [1\%] vs. unfractionated heparin 6 [1\%]; $\mathrm{p}=0.23$ ). No difference was noted for symptomatic intracranial hemorrhage between groups (4 [1\%] vs. 6 [1\%], respectively; $\mathrm{p}=0.55$ ); and the rate of major extracranial (gastrointestinal) bleeding was higher with enoxaparin than with unfractionated heparin $(7[1 \%]$ vs. $0 ; p=0.015)$. The study concluded that for patients with acute ischemic stroke, enoxaparin was preferable to unfractionated heparin for venous thromboembolism prophylaxis because of its superior clinical benefit to risk ratio and convenience of once daily administration.

\section{Transient Ischemic Attack Treatment}

Title: Effect of urgent treatment of transient ischemic attack and minor stroke on early recurrent stroke (EXPRESS study): a prospective population-based sequential comparison.

The risk of recurrent stroke is up to $10 \%$ in the week after a transient ischemic attack (TIA) or minor stroke [32]. This risk has been reported to be reduced by 80 to $90 \%$ by urgently initiating existing treatments such as antiplatelet aspirin or clopidogrel, anticoagulant, statin, and an ACE inhibitor [33], but in the absence of evidence for early existing treatment many health-care facilities have made little provision to offer such treatment. This study aimed to determine the effect of rapid implementation of such an existing treatment after TIA and minor stroke in patients who are not admitted directly to the hospital [34]. In this prospective before versus after (phase 1: April 1, 2002, to Sept 30, 2004 ; phase 2: Oct 1, 2004, to March 31, 2007) study of the effect of more urgent assessment and immediate treatment in the clinic, rather than in primary care, on all patients with TIA or minor stroke not admitted directly to a hospital. This study was nested within a population-based study of all TIA and stroke in a specific catchment area in southern England (Oxford Vascular Study; OXVASC). The primary outcome was the risk of stroke within 90 days of first seeking medical attention. Of the 1278 patients in OXVASC who presented with TIA or mild stroke (634 in phase 1 and 644 in phase 2), 607 were referred or presented directly to hospital, 620 were referred for outpatient assessment, and 51 were not referred. Of the outpatient referrals to the study clinic $(95 \%, n=591)$ baseline characteristics and delays in seeking medical attention were similar in both periods, but median delay to assessment in the study clinic fell from 3 (IQR 2-5) days in phase 1 to less than $1(0-3)$ day in phase $2(\mathrm{p}<0.0001)$, and median delay to first prescription of treatment fell from 20 (8-53) days to $1(0-3)$ day $(\mathrm{p}<0.0001)$. The 90 -day risk of recurrent stroke in the patients referred to the study clinic was $10.3 \%$ (32/310 patients) in phase 1 and $2.1 \%(6 / 281$ patients) in phase 2 (adjusted hazard ratio $0.20,95 \%$ CI $0.08-0.49 ; \mathrm{p}=0.0001$ ). This reduction in risk of recurrent stroke was independent of age and sex. Early initiation of existing treatment did not increase the risk of intracerebral hemorrhage or other bleeding. Thus early initiation of existing treatments after TIA or minor stroke was associated with an $80 \%$ reduction in the risk of early recurrent stroke. The beneficial results of early treatment in this study are an eye opener for the need for service provision and public education about TIA and minor stroke.

Title: Fast assessment of stroke and transient ischemic attack to prevent early recurrence (FASTER): a randomized controlled pilot trial. 
Patients with transient ischemic attack (TIA) or minor stroke are at high immediate risk of stroke. In this study 392 patients with TIA or minor stroke were randomly assigned within $24 \mathrm{~h}$ of symptom onset to clopidogrel $(300 \mathrm{mg}$ loading dose then $75 \mathrm{mg}$ daily; 198 patients) or placebo (194 patients), and simvastatin (40 mg daily; 199 patients) or placebo (193 patients) [35]. All patients were also given aspirin and were followed for 90 days. Analysis was by intention to treat. The primary outcome was total stroke (ischemic and hemorrhagic) within 90 days. Safety outcomes included hemorrhage related to clopidogrel and myositis related to simvastatin. The median time to stroke outcome was 1 day (range 0-62 days). The trial was stopped early due to a failure to recruit patients at the prespecified minimum enrolment rate because of increased use of statins. Some $14(7.1 \%)$ patients on clopidogrel had a stroke within 90 days compared with $21(10.8 \%)$ patients on placebo (risk ratio 0.7 [95\% CI
0.3-1.2]; absolute risk reduction $-3.8 \%$ [95\% CI -9.4 to 1.9]; $\mathrm{p}=0.19)$. Some $21(10.6 \%)$ patients on simvastatin had a stroke within 90 days compared with 14 (7.3\%) patients on placebo (risk ratio 1.3 [0.7-2.4]; absolute risk increase 3.3\% [-2.3 to 8.9]; $\mathrm{p}=0.25)$. There was no interaction between clopidogrel and simvastatin $(\mathrm{p}=0.64)$. Two patients on clopidogrel had intracranial hemorrhage compared with none on placebo (absolute risk increase $1.0 \%$ [-0.4 to 2.4]; $\mathrm{p}=0.5$ ). There was no difference between groups for the simvastatin safety outcomes. In conclusion, patients who are at a high risk of ischemic stroke immediately after TIA or minor stroke, the potential benefit of ischemic stroke reduction from combined aspirin and clopidogrel was not offset by their hemorrhagic risk. This study did not provide evidence of a benefit of simvastatin in this setting. A summary of these RCTs in Stroke Rehabilitation in 2007 is presented as Table 1.

Table 1. Summary of the Randomized Control Trials Undertaken in Stroke Rehabilitation in 2007

\begin{tabular}{|c|c|c|c|c|}
\hline References & $\begin{array}{l}\text { Time to Randomi- } \\
\text { zation Post-Stroke }\end{array}$ & Set-up & $\begin{array}{l}\text { Sample } \\
\text { Size }\end{array}$ & Main Findings \\
\hline \multicolumn{5}{|c|}{ Hyperlipidaemia and Stroke } \\
\hline [2] & $\begin{array}{l}\text { Within } 3 \text { days of acute } \\
\text { stroke }\end{array}$ & $\begin{array}{l}\text { Acute hospitali- } \\
\text { zation }\end{array}$ & 89 & $\begin{array}{l}\text { Statin withdrawal was associated with increased risk of } \\
\text { death or dependency at } 90 \text { days. Hence, this treatment } \\
\text { should be continued in the acute phase of ischemic stroke. }\end{array}$ \\
\hline [3] & - & $\begin{array}{l}\text { Out-patient set- } \\
\text { ting }\end{array}$ & 984 & $\begin{array}{l}\text { In middle-aged adults with an FRS of less than } 10 \% \text { and } \\
\text { evidence of sub-clinical atherosclerosis, rosuvastatin sig- } \\
\text { nificant reduced the rate of progression of maximum CIMT } \\
\text { over } 2 \text { years vs. placebo. }\end{array}$ \\
\hline \multicolumn{5}{|c|}{ Diabetes Mellitus and Stroke } \\
\hline [4] & - & $\begin{array}{l}\text { Out-patient set- } \\
\text { ting }\end{array}$ & 5238 & $\begin{array}{l}\text { Pioglitazone was effective in secondary not in primary } \\
\text { stroke prevention in patients with type } 2 \text { diabetes. }\end{array}$ \\
\hline [5] & $\begin{array}{l}\text { Within } 24 \text { hours of an } \\
\text { acute stroke }\end{array}$ & $\begin{array}{l}\text { Acute hospitali- } \\
\text { zation }\end{array}$ & 933 & $\begin{array}{l}\text { Despite significant reduction in the plasma glucose concen- } \\
\text { trations and blood pressure in the GKI group, this interven- } \\
\text { tion did not reduce death or disability rates. Thus maintain- } \\
\text { ing euglycaemia by GKI infusion may have a very small } \\
\text { effect in reducing death or disability, given that it could not } \\
\text { be seen in }>900 \text { subjects studied. }\end{array}$ \\
\hline \multicolumn{5}{|c|}{ Thrombolytic and Stroke } \\
\hline [8] & $\begin{array}{l}\text { Within } 3 \text { hours of an } \\
\text { acute stroke }\end{array}$ & $\begin{array}{l}\text { Acute hospitali- } \\
\text { zation }\end{array}$ & 24 & $\begin{array}{l}\text { Administration of UA appeared to be safe, decreased lipid } \\
\text { peroxidation, and prevented an early fall of UA in serum in } \\
\text { patients treated with rt-PA within } 3 \text { hours of stroke onset. } \\
\text { However, no beneficial clinical effect between the } 2 \text { groups } \\
\text { was noted. }\end{array}$ \\
\hline [10] & $\begin{array}{l}\text { Within } 24 \text { hours of an } \\
\text { acute stroke }\end{array}$ & $\begin{array}{l}\text { Acute hospitali- } \\
\text { zation }\end{array}$ & 120 & $\begin{array}{l}\text { The NEST-1 study indicated that infrared laser therapy was } \\
\text { a safe and effective treatment for ischemic stroke in hu- } \\
\text { mans when initiated within } 24 \text { hours of stroke onset. }\end{array}$ \\
\hline [11] & $\begin{array}{l}\text { Within } 6 \text { hours of an } \\
\text { acute stroke }\end{array}$ & $\begin{array}{l}\text { Acute hospitali- } \\
\text { zation }\end{array}$ & 114 & $\begin{array}{l}\text { The primary end point did not reach statistical significance. } \\
\text { However, the secondary analyses showed that intra-arterial } \\
\text { thrombolysis to have the potential to increase excellent } \\
\text { functional outcome. }\end{array}$ \\
\hline \multicolumn{5}{|c|}{ Antiplateltes and Stroke } \\
\hline [14] & $\leq 6$-months & $\begin{array}{l}\text { Out-patient set- } \\
\text { ting }\end{array}$ & 1068 & $\begin{array}{l}\text { This study showed that medium intensity oral anticoagula- } \\
\text { tion (target INR range } 2.0-3.0 \text { ) was equally effective as } \\
\text { aspirin for secondary prevention after transient ischemic } \\
\text { attack or minor stroke of arterial origin. However, in- } \\
\text { creased bleeding complications in the oral anticoagulation } \\
\text { group offset its protective effect against ischemic events. }\end{array}$ \\
\hline [16] & - & $\begin{array}{l}\text { Out-patient set- } \\
\text { ting }\end{array}$ & 973 & $\begin{array}{l}\text { This study suggests anticoagulation therapy benefits people } \\
\text { aged over } 75 \text { who have atrial fibrillation, unless there are } \\
\text { contraindications to its use or the patient decides that the } \\
\text { hemorrhagic risk outweighs its benefit. }\end{array}$ \\
\hline
\end{tabular}


(Table 1). Contd.....

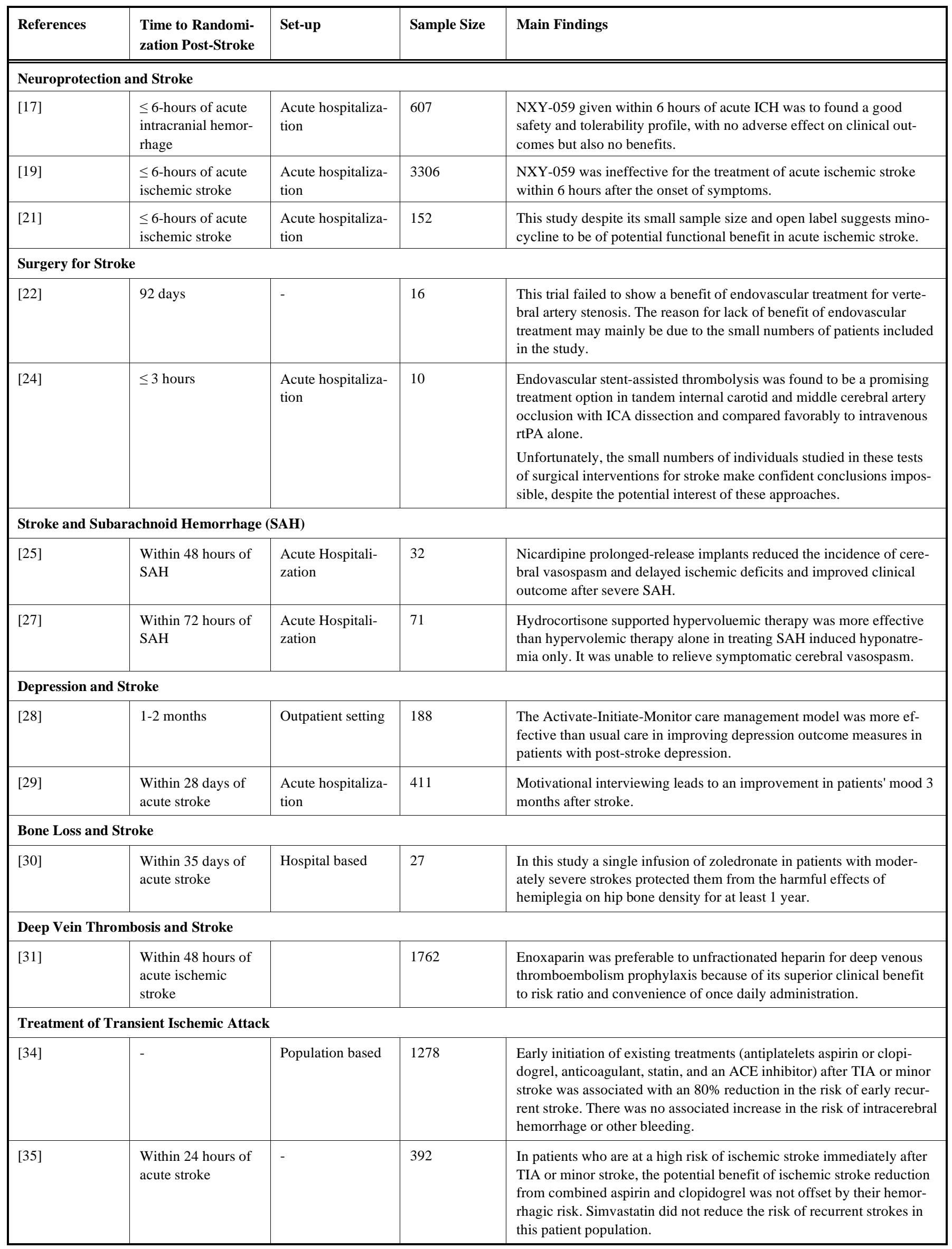




\section{CONCLUSIONS}

The following conclusions can be drawn from the studies summarized here: (1). Aggressive administration of glucosepotassium-insulin does not benefit acute stroke patients with hyperglycaemia. However, pioglitazone reduces the risk of recurrent strokes in Type 2 diabetic patients. (2) Nimodipine continues to be the prophylactic agent of choice in preventing vasospasm in patients with SAH because of the ease of oral administration. It should be given for a set time period of 3-weeks. (3) Warfarin is the agent of choice in elderly patients with atrial fibrillation living in the community. (4) The drug NXY-059 does not provide clinically significant neuroprotection in the clinical settings in which it has been tested. (5) Statins decrease morbidity and mortality in acute stroke patients already on statins, and are an effective agent in primary prevention of acute ischemic stroke. (6) Intraarterial Urokinase may be an effective thrombolytic agent instead of r-tPA in patients who present between 3 to 6 hours after an acute ischemic stroke. (7) Laser therapy is being developed as a non-pharmacological thrombolytic modality for treatment of stroke. (8) A single dose of Zoledronate, a bisphosphonate, helps maintain bone mineral density of the affected limbs and prevents fractures. (9) Low-molecular weight heparin (enoxaparin) prevents deep vein thrombosis as effectively as unfractionated heparin. (10) Stent-assisted endovascular thrombolysis is being suggested for internal carotid artery dissection with tandem internal carotid and middle cerebral artery occlusion and also in vertebral artery stenosis.

\section{REFERENCES}

[1] Shabanzadeh AP, Shuaib A, Wang CX. Simvastatin reduced ischemic brain injury and perfusion deficits in an embolic model of stroke. Brain Res 2005; 1042: 1-5.

[2] Blanco M, Nombela F, Castellanos M, et al. Statin treatment withdrawal in ischemic stroke: a controlled randomized study. Neurology 2007; 69: 904-10.

[3] Crouse JR 3rd, Raichlen JS, Riley WA, et al. METEOR Study Group. Effect of rosuvastatin on progression of carotid intimamedia thickness in low-risk individuals with subclinical atherosclerosis: the METEOR Trial. JAMA 2007; 297: 1344-53.

[4] Wilcox R, Bousser MG, Betteridge DJ, et al. PROactive Investigators. Effects of pioglitazone in patients with type 2 diabetes with or without previous stroke: results from PROactive (PROspective pioglitAzone Clinical Trial In macroVascular Events 04). Stroke 2007; 38: 865-73.

[5] Gray CS, Hildreth AJ, Sandercock PA, et al. GIST Trialists Collaboration. Glucose-potassium-insulin infusions in the management of post-stroke hyperglycaemia: the UK Glucose Insulin in Stroke Trial (GIST-UK). Lancet Neurol 2007; 6: 397-406.

[6] Romanos E, Planas AM, Amaro S, Chamorro A. Uric acid reduces brain damage and improves the benefits of rt-PA in a rat model of thromboembolic stroke. J Cereb Blood Flow Metab 2007; 27: 1420.

[7] Amaro S, Planas AM, Chamorro A. Uric acid administration in patients with acute stroke: a novel approach to neuroprotection. Expert Rev Neurother 2008; 8: 259-70.

[8] Amaro S, Soy D, Obach V, Cervera A, Planas AM, Chamorro A. A pilot study of dual treatment with recombinant tissue plasminogen activator and uric acid in acute ischemic stroke. Stroke 2007; 38: 2173-5.

[9] Yao H, Okada Y, Ibayashi S. Therapeutic time window for YAG laser-induced reperfusion of thrombotic stroke in hypertensive rats. Neuroreport 2002; 13: 1005-8.

[10] Lampl Y, Zivin JA, Fisher M, et al. Infrared laser therapy for ischemic stroke: a new treatment strategy: results of the NeuroThera Effectiveness and Safety Trial-1 (NEST-1). Stroke 2007; 38: 1843-9.
[11] Ogawa A, Mori E, Minematsu K, et al. MELT Japan Study Group Randomized trial of intraarterial infusion of urokinase within 6 hours of middle cerebral artery stroke: the middle cerebral artery embolism local fibrinolytic intervention trial (MELT) Japan. Stroke 2007; 38: 2633-9.

[12] Furlan A, Higashida R, Wechsler L, et al. Intra-arterial prourokinase for acute ischemic stroke. The PROACT II study: a randomized controlled trial. Prolyse in acute cerebral thromboembolism. JAMA 1999; 282: 2003-11.

[13] No authors listed. Secondary prevention in non-rheumatic atrial fibrillation after transient ischaemic attack or minor stroke. EAFT (European Atrial Fibrillation Trial) Study Group. Lancet 1993; 342: $1255-62$.

[14] The ESPRIT Study Group; Algra A. Medium intensity oral anticoagulants versus aspirin after cerebral ischaemia of arterial origin (ESPRIT): a randomised controlled trial. Lancet Neurol 2007; 6: 115-24.

[15] No authors listed. Secondary prevention in non-rheumatic atrial fibrillation after transient ischaemic attack or minor stroke. EAFT (European Atrial Fibrillation Trial) Study Group. Lancet 1993; 342: 1255-62.

[16] Mant J, Hobbs FD, Fletcher K, et al. BAFTA investigators; Midland Research Practices Network (MidReC). Warfarin versus aspirin for stroke prevention in an elderly community population with atrial fibrillation (the Birmingham Atrial Fibrillation Treatment of the Aged Study, BAFTA): a randomised controlled trial. Lancet 2007; 370: 493-503.

[17] Lyden PD, Shuaib A, Lees KR, et al. CHANT Trial Investigators. Safety and tolerability of NXY-059 for acute intracerebral hemorrhage: the CHANT Trial. Stroke 2007; 38: 2262-9.

[18] Lees KR, Zivin JA, Ashwood T, et al. Stroke-Acute Ischemic NXY Treatment (SAINT I) Trial Investigators. NXY-059 for acute ischemic stroke. N Engl J Med 2006; 354: 588-600.

[19] Shuaib A, Lees KR, Lyden P, et al. SAINT II Trial Investigators. NXY-059 for the treatment of acute ischemic stroke. N Engl J Med 2007; 357: 562-71

[20] Wang CX, Yang T, Noor R, Shuaib A. Delayed minocycline but not delayed mild hypothermia protects against embolic stroke. BMC Neurol 2002; 2: 2

[21] Lampl Y, Boaz M, Gilad R, et al. Minocycline treatment in acute stroke: an open-label, evaluator-blinded study. Neurology 2007; 69: 1404-10.

[22] Coward LJ, McCabe DJ, Ederle J, et al. CAVATAS Investigators Long-term outcome after angioplasty and stenting for symptomatic vertebral artery stenosis compared with medical treatment in the Carotid And Vertebral Artery Transluminal Angioplasty Study (CAVATAS): a randomized trial. Stroke 2007; 38: 1526-30.

[23] Lucas C, Moulin T, Deplanque D, Tatu L, Chavot D. Stroke patterns of internal carotid artery dissection in 40 patients. Stroke 1998; 29: 2646-8

[24] Lavallée PC, Mazighi M, Saint-Maurice JP, et al. Stent-assisted endovascular thrombolysis versus intravenous thrombolysis in internal carotid artery dissection with tandem internal carotid and middle cerebral artery occlusion. Stroke 2007; 38: 2270-4.

[25] Barth M, Capelle HH, Weidauer S, et al. Effect of nicardipine prolonged-release implants on cerebral vasospasm and clinical outcome after severe aneurysmal subarachnoid hemorrhage: a prospective, randomized, double-blind phase IIa study. Stroke 2007; 38: 330-6.

[26] Mori T, Katayama Y, Kawamata T, Hirayama T. Improved efficiency of hypervolemic therapy with inhibition of natriuresis by fludrocortisone in patients with aneurysmal subarachnoid hemorrhage. J Neurosurg 1999; 91: 947-52.

[27] Katayama Y, Haraoka J, Hirabayashi H, et al. A randomized controlled trial of hydrocortisone against hyponatremia in patients with aneurysmal subarachnoid hemorrhage. Stroke 2007; 38: 2373-5.

[28] Williams LS, Kroenke K, Bakas T, et al. Care management of poststroke depression: a randomized, controlled trial. Stroke 2007; 38: 998-1003.

[29] Watkins CL, Auton MF, Deans CF, et al. Motivational interviewing early after acute stroke: a randomized, controlled trial. Stroke 2007; 38: 1004-9.

[30] Poole KE, Loveridge N, Rose CM, Warburton EA, Reeve J. A single infusion of zoledronate prevents bone loss after stroke. Stroke 2007; 38: 1519-25. 
[31] Sherman DG, Albers GW, Bladin C, et al. PREVAIL Investigators. The efficacy and safety of enoxaparin versus unfractionated heparin for the prevention of venous thromboembolism after acute ischaemic stroke (PREVAIL Study): an open-label randomised comparison. Lancet 2007; 369: 1347-55.

[32] Coull AJ, Lovett JK, Rothwell PM. Oxford Vascular Study. Population based study of early risk of stroke after transient ischaemic attack or minor stroke: implications for public education and organization of services. BMJ 2004; 328: 6 .

[33] Goldstein LB, Bian J, Samsa GP, Bonito AJ, Lux LJ, Matchar DB. New transient ischemic attack and stroke: outpatient management by primary care physicians. Arch Intern Med 2000; 160: 2941-6.
[34] Rothwell PM, Giles MF, Chandratheva A, et al. Early use of Existing Preventive Strategies for Stroke (EXPRESS) study. Effect of urgent treatment of transient ischaemic attack and minor stroke on early recurrent stroke (EXPRESS study): a prospective populationbased sequential comparison. Lancet 2007; 370: 1432-42.

[35] Kennedy J, Hill MD, Ryckborst KJ, et al., FASTER Investigators. Fast assessment of stroke and transient ischaemic attack to prevent early recurrence (FASTER): a randomised controlled pilot trial. Lancet Neurol 2007; 6: 961-9.

(C) Rabadi and Blass; Licensee Bentham Open.

This is an open access article licensed under the terms of the Creative Commons Attribution Non-Commercial License (http://creativecommons.org/licenses/by-nc/3.0/) which permits unrestricted, non-commercial use, distribution and reproduction in any medium, provided the work is properly cited. 